\title{
Infección de Hypolobocera chilensis eigenmanni por metacercarias de Paragonimus mexicanus (= peruvianus) en el distrito de Condebamba (Cajamarca, Perú)
}

\author{
Paragonimus mexicanus (= peruvianus) metacercariae infection \\ Hypolobocera chilensis eigenmanni in Condebamba district \\ (Cajamarca, Perú)*
}

Alina Huiza F. (1), Yrma Espinoza B. (") y Carlos Sevilla A. ${ }^{(2)}$

\section{RESUMEN}

Cangrejos de rio Hypolobocera chilensis eigenmanni fueron colectados de acequias durante el año 1997 en estación seca (mayo a diciembre) en Chaquicocha, área que pertenece al distrito de Condebamba (departamento de Cajamarca) en la parte norte del Perú.

Ciento treinta y un cangrejos colectados fueron transportados al Laboratorio de Parasitología y examinados por disección, 27 de $131(20,6 \%)$ estaban infectados por metacercarias de Paragonimus mexicanus(=peruvianus). La intensidad de la infección fue de 1 a 5 en la mayoría de los casos $(81,5 \%)$ con un promedio de 4,85 por cangrejo. Estos datos son diferentes a los de estudios anteriores en la misma área donde fueron más altos, lo que indica una tendencia al decrecimiento del número de cangrejos infectados.

Palabras clave: cangrejos de río, metacercaria, Paragonimus.

\section{ABSTRACT}

Hypolobocera chilensis eigenmanni crabs were collected from small streams during the dry season, 1997 (May to December) in the Chaquicocha area belonging to the district of Condebamba (Department of Cajamarca) in the northern region of Peru.

One hundred thirty one crabs were collected, transported and examined in the Parasitology Laboratory by dissection, 27 out of $131(20,6 \%)$ were infected by Paragonimus mexicanus (=peruvianus) metace rcariae. The intensity of infection was from 1 to 5 in most of the cases $(81,5 \%)$ with an average of 4,85 per crab. These data are different from past studies in the same area with higher figures, indicating decreasing tendency in the number of infected crabs.

Key words: freshwater crab, metacercariae, Paragonimus.

\section{INTRODUCCIÓN}

El mayor número de casos de paragonimiosis humana diagnosticados en el país provienen del departamento de Cajamarca y principalmente del valle de Condebamba, segưn Ibáñez y Fernández. 1980. La infección

Protesor Asociado a Dedicación Exctusiva del D. A. de Microbiologia Médica y miembro del Instiluto de Medicina Tropical "Daniel A. Carrión de la Facultad de Medicina de la Univ. Nac. Mayor de San Marcos.

2 Protesor Auxiliar a T. P. 20 horas del D. A. de Microbiologia Médica de la Facultad de Medicina de la Univ. Nac. Mayor de San Marcos.

Este trabajo ha sido hnanciado por la Olicina General de Investigación de la UNMSM. P. de investigación 7010126 se adquiere por la costumbre de ingerir cangrejos crudos o mal cocidos, según Tantaleán y Hui ca, 1986.

En nuestro pais se ha demostrado îa presencia de metacercarias de Paragonimus mexicanus (sinónimo Paragonimus peruvianus) en cangrejos de la especie Hypolobocera chilensis eigenmanni según Cuba y cols., 1974 y Tantaleán y cols., 1974, considerada la principal especie que transmite al parásito. 
Varios estudios realizados en las décadas del $60,70 y$, en menor medida, a principios del 80 permitieron conocer y tener datos actualizados de la epidemiología de la paragonimosis en Cajamarca según Grados y cols. (1972), Yokogawa y cols. (1983) y Náquira y cols. (1976). Sin embargo, actualmente es escasa la información sobre paragonimosis en el departamento de Cajamarca. Para conocer cuán persistente es la fuente de infección humana y animal, nos propusimos estudiar la infección de los cangrejos (segundo hospedero intermediario) por metacercarias de Paragonimus del valle de Condebamba. Para ello se escogió la localidad de Chaquicocha, que en el pasado mostró casos autóctonos de paragonimosis humana, según Grados y cols. en 1972.

\section{MATERIAL Y MÉTODOS}

La recolección de los cangrejos se realizó en estación seca de mayo a diciembre de 1997. El lugar seleccionado para la captura de los cangrejos fue la localidad de Chaquicocha, distrito de Condebamba, provincia de Cajabamba, departamento de Cajamarca, un área rural en la cual se han reportado casos de paragonimosis humana, según Grados y cols. (1972).

Ciento treinta y un cangrejos fueron colectados de las oquedades de los canales de agua de río, que son utilizados para regar los cultivos.

Cada uno de los cangrejos recolectados fue examinado macroscópicamente; se registró el nombre de la especie, sexo, tamaño y peso. Los cangrejos fueron transportados vivos $y$ examinados en el laboratorio del Instituto de Medicina Tropical "Daniel A. Carrión"; sin embargo, los especímenes que resultaron muertos en la captura o a pocas horas después de ella, fueron examinados in situ. Para determinar si los órganos estaban diferencialmente parasitados, se realizó una disección detallada de los cangrejos.

Luego del recuento de las metacercarias encontradas, éstas fueron inoculadas en gatos domésticos para estudios posteriores.

\section{RESULTADOS}

Los cangrejos recolectados fueron diseccionados cuidadosamente, y se pudo observar que el único órgano parasitado era el hepatopáncreas.

De los 131 cangrejos colectados, 27 $(20,6 \%)$ estuvieron parasitados con metacercarias. La intensidad de la infección, que representa el número de metacercarias por cangrejo parasitado, fue de 4,85 (131/27). Asimismo, la frecuencia del número de metacercarias por cangrejo que predominó fue de 1 a $5 ; 22$ de 27 cangrejos $(81,5 \%)$ tuvieron más de 1 pero menos de 5 metacercarias (Tabla 1). El porcentaje de machos parasitados $(25,0 \%)$ fue mayor al de hembras $(17,7 \%)$. Además, se observó una correlación positiva entre el tamaño de los cangrejos y su parasitismo por metacercarias (Tabla 2). Las características morfológicas de las metacercarias fueron observadas in vivo correspondiendo la totalidad a formas típicas de metacercarias de Paragonimus mexicanus (Miyasaki y col. 1971).

La más alta proporción de cangrejos parasitados fue encontrada en el mes de junio $(29,2 \%)$, seguida de los meses de diciembre $(22,6 \%)$ y mayo $(20,7 \%)$, setiembre $(13,8 \%)$ y octubre $(16,6 \%)$.

Todos los cangrejos colectados (79 hembras y 52 machos) fueron de la misma especie Hypolobocera chilensis eigenmanni (Mayta y Uyema, 980 ). 
Tabla ㅅ.. 1

Número de metacercarias de $P$. mexicanus (=peruvianus) encontrado en los cangrejos de río

\begin{tabular}{cccc}
\hline N. ${ }^{\circ}$ de Metacercarias & Macho & Hembra & Total \\
\hline 1 & 9 & 6 & 15 \\
2 & 0 & 3 & 3 \\
4 & 1 & 2 & 3 \\
5 & 0 & 1 & 1 \\
8 & 1 & 0 & 1 \\
0 & 1 & 0 & 1 \\
10 & 0 & 1 & 1 \\
17 & 1 & 0 & 1 \\
55 & 0 & 1 & 1 \\
\hline Total & 13 & 14 & 27 \\
\hline
\end{tabular}

Tabla N.@2

Correlación dei tamaño del cangrejo parasitado con metacercarias de $P$. mexicanus (=peruvianus)

\begin{tabular}{cccc}
\hline Tamaño & Macho & Hembra & Total \\
\hline $4,0-4,9$ & 2 & 0 & 2 \\
$5,0-5,9$ & 3 & 1 & 4 \\
$6,0-6,9$ & 5 & 1 & 6 \\
$7,0-7,9$ & 3 & 4 & 7 \\
$8,0-8,9$ & 0 & 8 & 8 \\
\hline Total & 13 & 14 & 27 \\
\hline
\end{tabular}




\section{DISCUSIÓN}

La presencia de cangrejos parasitados con metacercarias de Paragonumus mexicumus en el valle de Condebamba demuestra la posibilidad de la infección humana. En estudios precedentes se han comunicado varios cásos de paragonimosis humana en el valle de Condebamba (Yokogawa y cols., 1983), una de cuyas localidades es Chaquicocha. La infección humana se mantiene por la costumbre de comer cangrejos crudos o insuficientemente cocidos (Graios y cols. 1972: Tintaleán y (Huiza, 1986). Si consideramos que el $20.6 \%$ de los cangrejos colectados por nosotros estuvieron pilrasitidos, existe una probalsilidad de $1: 5$ a adquirit la infección.

El porcentaje de cangrejos infechados en nuestra investigación es menor al reportado por orros autores como Cuba y cols. en 1974. Grados y cols. en 1972, Ibáñez y licinández. en 1980 y Tantaleán y cols. en 1974, en la misma zona estudiada. En el Valle de Condebamba Tantaleán y cols en 1974 encontraron que el $72,4 \%$ de los 69 Pseudothelphusa chilensis ( = Hypolobocera chilensis eigenmanni) estuvieron parasitados por $P$. mexicanus mientras que on Chaquicocha Cuba y cols. en 1974 encontraron que el $69,7 \%$ de 43 P. chilensis estaban parasitados con el mismo parásito, y Miyazaki y cols. en 1978 encontraron el $89.5 \%$ de $19 P$. chilensis. Esta tendencia decreciente de la infección de cangrejos por metacercarias en el valle de Condebamba ha sido sistemáticamente evaluada por. Huiza y Tantaleán en 1997 para los años 1993, 1994, 1995, cuyas tasas de infección fueron 34,7\%, $18,7 \%$ y $10,0 \%$ respectivamente. Simultáneamente al menor número de cangrejos parasitados. también se ha observado la disminución en la intensidad de la infección la cual ha variado de 26 (Tantaleán y cols., 1974) y 10,33 (Cuba y cols., 1974) en el año 1974 a 9,4; 4,5 y 1,5 en los años 1993, 1994 y 1995, respectivamente (Huiza y Tantaleán, 1997). Nuestro resultado de 4,85 para la intensidad de infección está más cercano a los obtenidos en los últimos años, lo cual confirmaría el descenso del parasitismo de cangrejos por Paragonimus. Estos resultados indican una modificación de los factores que determinan la transmisión de la infección.

En la misma zona de captura de los cangrejos sólo pudimos colectirr 2 caracoles Aroapyrgus sp. (datos no mostralos), probable primer hospedero intermediario de $P$. mexicanus (Malek y cols. 1985), lo que nos permite sugerir que la disminución de la pohlación de caricoles explicaría la baja tasa cle infestación en los cangrejos. Quedan por conocerse las alteraciones ecológicas producidas en el área estudiada que han conducido a esta situación, pues podría corresponder a procesus cíclicos ambientales.

Este cstudio indica que persiste la paliagonimosis en el valle de Condebambal, aunque las lassas cle prevaliencia de infección sean monores que lat de décaldas pasáldas. Es importante conocer los factores que han conclirrido a wita nueva situación de la paragonimosis, por lo yue sería apropiado intensilïar los estudios sobre la infección de los hospederos intermediarios, los reservorios y la previlencia en población humana.

\section{CONCLUSIONES}

Los canglejos de río Hypolobocera chilensis eigenmanni, recolectados en el valle de Condebamba (Chaquicocha) en el año 1997, se encontraron infectados en un 20,6\% $(27 / 131)$, lo cual favorecería la posibilidad de infección tanto humana como de animales silvestres y domésticos.

En la actualidad el valle de Condebamba debe seguir siendo considerado zona endémica de paragonimosis, y de riesgo de la infección humana que puede darse por la posibilidad $(1: 5)$ de ingerir un cangrejo positivo. 
Hay que considerar en el diagnóstico clínico diferencial a los pacientes provenientes de estas zonas con sintomatología similar a tuberculosis pulmonar con baciloscopía negativa y con el probable antecedente de haber ingerido cangrejos de río crudos o mal cocidos.

\section{AGRADECIMIENTOS}

Al doctor César Náquira, por la revisión y crítica del artículo; al Profesor William Cornejo, por ayudarnos en la redacción; al señor $A$. Medina, por su colaboración en la captura de los cangrejos.

\section{LITERATURA CITADA}

Cuba, C., Ibáñez N., Fernández E. y Miranda H. 1974. Paragonimus y Paragonimiasis en el Norte peruano. Infección natural de Pseudothelphusa chilensis por metacercarias de Paragonimus 1899. Rev. Peruana Biol.; 1: 128-135.

Grados, O., Cuba C., Morales N. y Mazabel. 1972. Epidemiología de la Paragonimiasis en el Perú. Arch. Per. Pat. Clin.; 26: 33-54.

Huiza, A. y Tantaleán M. 1997. La infección de cangrejos por metacercarias de Paragonimus en el valle de Condebamba, departamento de Cajamarca. Bol. Peruano Parasitol. 13:66-71.

Ibáñez, N. y Fernández E. 1980. Actual state of the Paragonimiasis in Peru. Bol. Peruano Parasitol. 2: 12-18.

Malek, E. Ibáñez N. and Guerra A. 1985. Description of redia and cercaria from experimentally infected Aroapyrgus colombiensis of Condebamba valley, Perú. J. Parasit.; 71: 253-256.

Mayta, R. y Uyema N. 1980. Cangrejos de importancia sanitaria en el Perú. Anales Científicos UNA, 18:75-81

Miyazaki, Y., Ibáñez N. y Miranda H. 1971 Studies on the Metacercariae of Paragonimus peruvianus (Trematoda, Troglotrematidae). Jap. J. Parasitol.; 20: 425-430.

Miyazaki, I., Kifune T., Habe S. y Uyema N 1978. Reports of Fukuoka University Scientific Expedition to Peru, 1976; Occasional Públication N. ${ }^{\circ}$ pp. 28.

Náquira, C., Delgado E., Náquira F., Elliot A. y Tantaleán M. 1976. La paragonimiasis en la población de los distritos de San Juan y Magdalena (departamento de Cajamarca), 1971 BIOTA; 11:23-33.

Tantaleán, M. y Huiza A. 1986. Paragonimus y Paragonimiasis en el Perú. (Segunda parte). Bol. Med. Trop; 5: 72-80.

Tantaleán, M., Huiza A. y Delgado E. 1974. La infección de cangrejos procedentes del valle de Condebamba (Cajamarca) por metacercarias de Paragonimus. Rev. Peruana Biol.; 1: 192-193.

Yokogawa, M., Inatomi S., Tsuji M., Kojima S., Kobayashi M., Hata H., Miranda H., Ibáñez N. y Rumber J. 1983. Pathobiological studies on Paragonimiasis in Peru and Ecuador. Chiba University. 